\title{
Attitudes of the Lebanese public regarding disclosure of serious illness
}

Salim M Adib and Ghassan N Hamadeh Kuwait University, Kuwait and the American University of Beirut, Lebanon respectively

\begin{abstract}
Objectives-To measure the preference regarding disclosure of a serious diagnosis, and its determinants, of the Lebanese public.

Design and setting-Non-random sample survey of 400 persons interviewed in health care facilities in Beirut in 1995.

Results-Forty-two per cent of respondents generally preferred truth not to be disclosed directly to patients. Preference for disclosure was associated with younger age, better education and tendency to rapport-building with physicians. There were no meaningful associations between place of residence (urban/rural), level of religious practice, or religious affiliation, and preference for disclosure.

Conclusions-Under one plausible interpretation, this survey suggests that the expectation for concealment will decrease as the advantage of knowledge in better coping with disease is understood by an increasingly better educated public, and that the Lebanese public will increasingly come to expect direct and full disclosure of serious diagnoses.

(Fournal of Medical Ethics 1999;25:399-403)
\end{abstract}

Keywords: Ethics; disclosure; survey; attitudes; Lebanon; Middle-East

\section{Introduction}

In Lebanon, physicians often withhold information on the true nature of a patient's diagnosis and prognosis, or give her false hopes about the illness when it is serious or terminal. A recent survey of a sample of 212 physicians in the capital, Beirut, showed that $53 \%$ would usually avoid disclosure of cancer diagnosis directly to patients. Remarkably, $59 \%$ of the entire group surveyed, including both those who usually do and do not disclose the diagnosis, agreed that they would change their policy should the public's expectations change. ${ }^{1}$ This finding indicated that the common perception of what the Lebanese patient really expects from her physician, though vague, nevertheless profoundly determines the physician's choice to disclose or withhold the truth. The extent of the preference not to be told of a serious diagnosis in the Lebanese public has so far never been formally assessed. This survey of preferences among outpatients and visitors at the American University of Beirut Medical Center (AUBMC) represents the first attempt to discover such attitudes.

\section{Background}

The Republic of Lebanon is a parliamentarian pluralistic democracy located on the eastern shores of the Mediterranean. This small country $\left(10,452 \mathrm{~km}^{2}\right.$ or $\left.4,035 \mathrm{sq} \mathrm{m}\right)$ is bordered by Syria to the north and east and by Israel to the south. The Lebanese population is relatively young: $60.5 \%$ are under the age of $30 .^{2}$ Almost two-thirds of Lebanese are Muslims, and the rest mostly Christians. The majority ( $60 \%-70 \%$ ) of the Lebanese population, estimated at 3.5 million, lives in the "Greater Beirut" area in and around Beirut, the centre of the economic and cultural life of the country. The official language in the country is Arabic. While the sociological structure is still largely traditional and patriarchal, there are marked differences between rural and urban areas, and between more or less educated Lebanese. All secondary-schooled Lebanese ( $60 \%$ of the population by some estimates) are fluent in either French and/or English, and are fairly exposed to non-traditional ideas and lifestyles. There are no restrictions on the amount of contacts that the Lebanese population can make (and the information they can obtain) either by travelling abroad or by interacting with foreign visitors at home. The Lebanese medical services are among the most sophisticated and up-to-date in the Arab-speaking countries of the eastern Mediterranean area. All major tertiary care centres in Lebanon are currently located within the Greater Beirut area.The American University of Beirut Medical Center, the health facility where this survey was conducted, was established in the early part of the 20th century. It is the largest tertiary health care centre in Lebanon, and it receives patients from all parts of the country.

The current Lebanese Code of Medical Ethics, enacted in 1995, does not mandate that physicians 
should inform patients of their diagnosis if such a disclosure is deemed by the doctor to be "deleterious to the patient's recovery". On the other hand, the law clearly states that patients have the right to know all their options regarding treatment, and to refuse any or all of those options. ${ }^{3}$ The apparent discrepancy between these two statements has left most Lebanese doctors uncertain as to the "right" approach to doctor-patient communication in cases of serious or terminal disease. Consequently, a majority usually opts for what is thought to be the culturally acceptable norm: that is withholding disclosure from the patient and disclosing instead to close family members. ${ }^{1}$ Problems arise, however, when mutilating therapies are proposed: how can a blinded patient exert her freedom of choice if she ignores the seriousness of her diagnosis? And how are the patient's prospects affected when a shattering reality is finally disclosed almost in extremis? These are some of the dilemmas which prompted the debate on disclosure, and finally won the day for it, in many "Western" societies. ${ }^{4}$ This paper aims to start the same kind of debate in Lebanon, and by extension in areas with similar cultural features and sociological structures. In this debate, the evolving opinions and expectations of the public must be taken into account. By revealing those expectations, we aim to help physicians adopt those practices most in tune with patients' freedom of choice as well as hope for recovery.

\section{Methods}

SELECTION OF PARTICIPANTS

Three groups of individuals were interviewed for the purposes of this survey on five consecutive weekdays in July 1995. These groups were:

1) The first 100 previously diagnosed cancer outpatients (COP) attending a specialised cancer clinic at AUBMC.

2) The first 100 outpatients (OP) attending all other clinics for any health-related problem during the same period.

3) The first 300 "healthy" visitors who could be located in waiting areas during the same period.

Some respondents who had mistakenly identified themselves as outpatients were subsequently re-classified as "healthy" visitors upon review of their responses. Two incomplete interviews were later discarded.

\section{INFORMED CONSENT}

The survey proposal was reviewed and approved by the Human Subject Board of the AUB Faculty of Medicine prior to implementation. The aim of the interview was clearly stated to all potential participants who were totally free to opt out of the study at any moment. Only a handful of persong approached actually preferred not to participate

INTERVIEW PROCEDURES AND TOOLS

Face-to-face interviews were conducted by medî cal interns and junior nurses. Special care was taken to avoid other people overhearing the inter view. Whenever possible, the process was core ducted in an empty room or cubicle. A slight modified version of a questionnaire developed bis Sardell and Trierweiller in $1993^{5}$ was used for this study. It consists of items covering the following large categories:

1) Socio-cultural and demographic variables; health status, and current health complaint. O

2) A series of statements with a Likert visua scale, eliciting participants' attitudes, belies and expectations regarding disclosure of seri ous or terminal diagnosis.

\section{STATISTICAL METHODS}

Factor analysis was used to create two indices ou⿳亠二口犬 of the list of attitude statements. The first inde "preference to sickness disclosure", was used $\$$ an outcome variable and included five question $\overrightarrow{\vec{\varepsilon}}$ The second one, "tendency to building rapport with physician" (rapport-building), included twg questions. To be included in an index, variables had to load in factor analysis with a principa component $\geqslant \pm 0.60$. Statements included in the indices are presented in the appendix. Some other. statements failed to show any variability and were ultimately "pruned" to simplify the analysis. Fre quencies of the outcome and various independerg variables were computed. The association of seve independent variables with the outcome wa assessed separately, controlling for sex and aga These variables were: health status (three categories), occupation (four categories), education (two levels), area of residence (Greater Beirut, urba@ province, rural province), religious denominatio (Muslim or not), intensity of religious practice (three levels), and rapport-building (two levels Associations were presented as odds-ratios (OR) with corresponding $95 \%$ confidence intervas ( $95 \%$ CI). The OR here represents the odds off higher preference for disclosure in a give category compared to the same in a reference cae egory. Results are significant when the $95 \%$ Cf does not include the unity. All data were entered and analyzed using the Statistical Package fo Social Sciences (SPSS) on Windows. 
Table 1 Preference for disclosure of serious sickness in the Lebanese public by demographic variables

\begin{tabular}{lllll}
\hline & Lower & Higher & Total & p-value \\
\hline n (\%) & $207(41.6)$ & $291(56.8)$ & $498(100)$ & \\
Sex (n, \%) & & & & \\
$\quad$ Males & $73(35.3)$ & $138(47.4)$ & $211(42.4)$ & $<0.01$ \\
$\quad$ Females & $134(64.7)$ & $153(52.6)$ & $287(57.6)$ & \\
Age (mean years, SD) & $41.2(14.1)$ & $33.5(13.0)$ & $36.7(14.0)$ & $<0.01$ \\
\hline
\end{tabular}

*Using the X-square test for "sex" and the Student t-test for "age"

\section{Results}

A total of 498 persons were interviewed: 88 cancer outpatients $(17.7 \%), 99$ non-cancer patients $(19.9 \%)$ and 311 "healthy" persons visiting or accompanying patients $(62.4 \%)$. The mean age of participants was $37 \pm 14$ years. Males were $42 \%$ of the group and females $58 \%$. Participants were mostly Muslim (62\%) residents of Greater Beirut $(68 \%)$, and with a secondary education or more $(60 \%)$. Most of them (63\%) indicated strong levels of religious practice. Lower tendency to rapport-building with the physician was found in $59.2 \%$ of respondents, while lower preference for disclosure of cancer diagnosis was found in $41.6 \%$. Overall about $42 \%$ of the total 498 respondents indicated a preference for nondisclosure of serious or terminal diagnosis.

As shown in table 1, male respondents were significantly more likely to have a higher preference for disclosure than females. There were also significant age differences between those with higher preference for disclosure (average age 33.5 years) and those with lower preference (average age 41.2 years). Further analysis, however, showed that the sex differential in disclosure preference was not significant in those aged 37 years (total average age for the group) or less. Age was significantly correlated with other dependent variables. Younger respondents were also better educated, less religious, and more likely to build a rapport with their physicians. In view of its significant effect on the outcome, age was used as a control variable in the subsequent analysis.

As presented in table 2, higher preference for disclosure was significantly lower among those with only primary or with no education compared to those with secondary education and more (OR $=0.60)$. No significant differences in disclosure preference were found by occupation, except when comparing housewives to those in professional or managerial jobs. The latter were significantly more likely to prefer disclosure than the former $(O R=0.40)$. There were no significant differences in preference associated with either usual place of residence or level of religious practice. Non-Muslims, a heterogeneous group which included Christians ( $24 \%$ of all participants), Druze $(2 \%)$ and those with unstated religious affiliation (11\%), had significantly higher preference for disclosure than the $63 \%$ self-declared Muslims $(O R=1.52)$. However, that finding was heavily confounded by education. Almost half of the "Muslim" category (49.7\%) in the sample

Table 2 Determinants of preference for disclosure of serious sickness in the Lebanese public

\begin{tabular}{|c|c|c|c|c|}
\hline & Lower preference & Higher preference & Total & OR $(95 \% C I) \#$ \\
\hline n (\%) & $207(41.6)$ & $291(56.8)$ & $498(100)$ & - \\
\hline \multicolumn{5}{|l|}{ Education } \\
\hline None/Primary & $121(58.5)$ & $76(26.1)$ & $197(39.6)$ & $0.60(0.48 ; 0.74)$ \\
\hline Secondary or more & $86(41.5)$ & $215(73.9)$ & $301(60.4)$ & reference \\
\hline \multicolumn{5}{|l|}{ Occupation } \\
\hline Unemployed/retired/student & $26(12.6)$ & $73(25.1)$ & 99 (19.9) & $1.26(0.82 ; 1.92)$ \\
\hline Housewife & $112(54.1)$ & $73(25.1)$ & $185(37.1)$ & $0.40(0.17 ; 0.94)$ \\
\hline Manual jobs & $30(14.5)$ & $34(11.7)$ & $64(12.9)$ & $0.95(0.60 ; 1.50)$ \\
\hline Professional/managerial jobs & $39(18.8)$ & $111(38.1)$ & $150(30.1)$ & reference \\
\hline \multicolumn{5}{|l|}{ Residence } \\
\hline Greater Beirut & $128(61.8)$ & $209(71.8)$ & $337(67.7)$ & reference \\
\hline Urban province & $32(15.5)$ & $37(12.7)$ & $69(13.9)$ & $0.84(0.58 ; 1.22)$ \\
\hline Rural province & $47(22.7)$ & $45(15.5)$ & $92(18.5)$ & $0.86(0.61 ; 1.21)$ \\
\hline \multicolumn{5}{|l|}{ Religious affiliation } \\
\hline Muslim & $152(73.4)$ & $160(55.0)$ & $312(62.7)$ & reference \\
\hline Non-Muslim & $55(26.6)$ & $131(45.0)$ & $186(37.3)$ & $1.52(1.24 ; 1.86)$ \\
\hline \multicolumn{5}{|l|}{ Religious practice } \\
\hline Low & $11(5.3)$ & $30(10.3)$ & $41(8.2)$ & $1.19(0.72 ; 2.12)$ \\
\hline Medium & $40(19.3)$ & $105(36.1)$ & $145(29.1)$ & $1.31(0.92 ; 1.86)$ \\
\hline High & $156(75.4)$ & $156(53.6)$ & $312(62.7)$ & reference \\
\hline \multicolumn{5}{|l|}{ Health status } \\
\hline Cancer out-patients & $27(13.0)$ & $61(21.0)$ & $88(17.7)$ & $1.51(1.05 ; 2.16)$ \\
\hline Non-cancer out-patients & $50(24.2)$ & 49 (16.8) & $99(19.9)$ & $0.98(0.69 ; 1.40)$ \\
\hline Healthy individuals & $130(62.8)$ & $181(62.2)$ & $311(62.4)$ & reference \\
\hline \multicolumn{5}{|l|}{ Rapport-building } \\
\hline Lower & $153(73.9)$ & $142(48.8)$ & $295(59.2)$ & $0.63(0.51 ; 0.77)$ \\
\hline Higher & $54(26.1)$ & $149(51.2)$ & $203(40.8)$ & reference \\
\hline
\end{tabular}

\#Age-adjusted. 
described themselves as having had a primary education or less, while only $22.6 \%$ reported the same in the catchall "non-Muslim" category ( $p<$ 0.01 ). The same differential in education remained after a more conservative comparison was conducted using only self-described Muslims and Christians.

Cancer outpatients were significantly more likely to prefer disclosure compared to healthy participants $(O R=1.51)$. In contrast, non-cancer outpatients did not differ in preference from the healthy group. Those with a higher rapportbuilding tendency were also significantly more likely to have higher preference for disclosure than those with lower rapport-building tendency.

\section{Discussion}

Participants in this survey represent the Lebanese population in many aspects, such as the relatively young average age, the predominance of residence within Greater Beirut, and the Muslim affiliation of the majority. However, participants may be more heavily drawn from lower socio-economic groups, as more affluent or better educated patients may consult physicians outside the frame of the less expensive AUB outpatient clinics. This potential bias may have resulted in an underestimation of the higher preference for disclosure of serious or terminal diagnosis. Nevertheless, and despite this effect, it was still found that more than half those interviewed indicated a somewhat high preference to be told the truth about the diagnosis, extension and prognosis of serious or terminal disease.

Preference for disclosure was associated with health status: cancer patients were more likely to prefer disclosure compared to all others. However, this finding may be biased by those patients' previous knowledge of their diagnosis, which may have already helped them perform the mental adjustment needed to cope with further adverse events. The preference for disclosure was significantly associated with younger age regardless of sex. Older women, especially self-described housewives, were less likely to prefer disclosure than older men. Better educated and rapportbuilding participants were generally more likely to prefer disclosure than otherwise. These characteristics were associated with the outcome even after controlling for age. There were no associations between place of residence, level of religious practice, or religious affiliation and preferences for disclosure, once education was taken into account.

Overall, this analysis showed that $42 \%$ of Lebanese respondents generally preferred truth about serious and terminal illness not to be disclosed to patients. Preference for disclosure was als assessed in recent years in other Arab countries. $\mathrm{A}$ survey conducted in 1995 in a non-random sank ple in the United Arab Emirates (UAE) indicate that about $40 \%$ did not favour disclosure terminal diagnosis under any circumstances. Tha proportion decreased only slightly when the respondent was the patient in question. Youngeth better educated respondents were significant $\frac{\vec{B}}{\vec{B}}$ more inclined to prefer disclosure than older ones. ${ }^{6}$ A 1985 non-random survey of healthy Says dis revealed that $31 \%$ preferred a cancer diagnosis not to be disclosed directly to the patient. There too, the preference for disclosure was associated with younger age, and with family history of cancer. ${ }^{7}$ In Greece, a country sharing easterf Mediterranean heritage with Lebanon, a 1992 survey of healthy subjects revealed that about $35 \%$ of respondents thought that patients with termin illness should not be told the truth. As if Lebanon, UAE and Saudi Arabia, this preferencis was associated with older age. Also, as with Lebanon, higher preference for truth was associate with professional status but not with religious practice. ${ }^{8}$ In none of the studies reviewed did gero der emerge as a factor significantly associated wit disclosure preference.

Preference for concealment is thought to be the prevalent attitude in traditional cultures where "paternalistic/beneficent" attitudes of physiciar predominate over the respect for the "autonomy rights of patients. ${ }^{8-10}$ However, surveys fro Lebanon and other countries with overlappinig cultural characters indicate that opinions on the issue are actually very widely distributed. Moreer ver, higher preference for truth is consistent associated with younger age, better education and better professional achievement. Under one plau疋 sible interpretation, these findings indicate th denying information "is more a historical than cultural phenomenon". ${ }^{10}$ With such an interpret. ation, the expectation for concealment can be expected to decrease as the effectiveness of medi cine is demonstrated, and the advantage knowledge in better coping with and resisting disw ease is understood, by an increasingly better edu cated public. Physicians who are currently faceg with the dilemma of disclosing a serious diagnosis to patients in Lebanon and countries with similar cultures might still feel the need to tailor theig decision according to the socio-demographic prof files of patients. Great care should still be taken to respect the openly declared or implicit preference्ध of patients who do not want to be told the truth. This used to be the case in "Western" culturef about 20 years ago." But the dynamics which eventually rendered truth-telling a moral absolut 
in those cultures are also active in Lebanon. Given the above interpretation, it is very likely that the expectation of full disclosure in the Lebanese public, and its full implementation by Lebanese physicians, will soon reach "Western" levels. Monitoring of changes must be conducted within the coming few years to ascertain the accuracy of these predictions.

\section{Appendix}

QUESTIONS INCLUDED IN PREFERENCE INDICES

PREFERENCE TO KNOW THE TRUTH INDEX (OUTCOME INDEX)

1) I want to know the truth about my disease even if it is incurable. $(\alpha=0.61)$

2) I prefer a diagnosis of cancer to be disclosed to me first. $(\alpha=67)$

3) I prefer to be told how far a malignancy has spread in my body. $(\alpha=0.60)$

4) I prefer to receive all information regarding my disease, good or bad. ( $\alpha=0.77)$

5) The doctor must call a disease by its name, even if it is cancer. $(\alpha=0.77)$

RAPPORT-BUILDING WITH THE PHYSICIAN

1) I prefer to share my feelings, fears and hopes with my doctor. $(\alpha=0.70)$

2) I like it when my doctor discusses with me issues not related to my sickness, such as work, family or school issues. $(\alpha=0.71)$

\section{Acknowledgments}

Thanks are due to Ms Frida Hassoun, Dr Beatrice Khater and Dr Camille Aizarani for their efforts in data collection. This study was supported in part by grant No DCU-27110-686029 from the American University of Beirut Medical Practice Plan Fund.
Salim M Adib, MD, DrPH, is Assistant Professor of Community Medicine in the Department of Community Medicine, Kuwait University, Kuwait. Ghassan $N$ Hamadeh, $M D$, is Associate Professor of Family Medicine in the Department of Family Medicine, American University of Beirut, Lebanon.

\section{References}

1 Hamadeh GN, Adib SM. Truth telling to cancer patients among Lebanese physicians. Social Science and Medicine 1998;47:1289-94.

2 Directorate for Central Statistics, Office of the Prime Minister. Lebanese statistics, 1994. Beirut, Lebanon: Directorate for Central Statistics, 1995.

3 Lebanese Code of Medical Ethics. Decree 24. Fournal Officiel Libanais 1995 May.

4 Ad Hoc Committee on Medical Ethics. American Physicians' ethics manual. Part 1: history of medical ethics, the physician and the patient, the physician's relationship to other physicians, the physician and society. Annals of Internal Medicine 1984;101: 129-37.

5 Sardell AN,Trierweiller SJ. Disclosing the cancer diagnosis. Cancer 1993;72:3355-65.

6 Harrison A, Al-Saadi AMH, Al-Kaabi ASO, Al-Kaabi MRS, Al-Bedwawi SSM, et al. Should doctors inform terminally ill patients? The opinions of nationals and doctors in the United Arab Emirates. Fournal of Medical Ethics 1997;23:101-7.

7 Bedikian AY, Thompson SE. Saudi community attitude towards cancer. Annals of Saudi Medicine 1985;3:161-6.

8 Dalla-Vorgia P, Katsouyanni TN, Touloumi G, Drogari P, Koutselinis A. Attitudes of a Mediterranean population to the truth-telling issue. Fournal of Medical Ethics 1992;18:67-74.

9 Brusamolino E, Surbone A. Telling the truth to the patient with cancer. A cross-cultural dialogue. Annals of the New York Academy of Sciences 1997;809:411-21.

10 Pellegrino ED. Is truth-telling to patients a cultural artifact? fournal of the American Medical Association 1992;268:1734-5.

11 Cassileth BR, Zupkis RV, Sutton-Smith K, March V. Information and participation preferences among cancer patients. Annals of Internal Medicine 1980;92:832-6.

\section{News and notes \\ Ethics Education in Medical Schools}

An international conference on Ethics Education in Medical Schools will be held in Eilat, Israel from February 13 - 16, 2000.
For further information please contact the secretariat at: POB 574, Jerusalem, Israel 91004. Tel: 972-26520574; fax: 972-2-6520558. 